\title{
Simulation tools for pinched-electron-beam radiographic diodes
}

\author{
Stanley Humphries \\ Field Precision, P.O. Box 13595, Albuquerque, New Mexico 87192, USA \\ Thaddeus Orzechowski \\ Lawrence Livermore National Laboratory, Livermore, California 94550, USA
}

(Received 22 December 2005; published 21 February 2006)

\begin{abstract}
We describe capabilities of an integrated software suite to simulate pinched-electron-beam diodes for pulsed radiography. In contrast to other reported work using particle-in-cell methods, we employ a raytracing code (TRAK) with advanced capabilities for modeling beam-generated magnetic fields. Ray tracing is a direct approach to a steady-state solution and involves less work than a particle-in-cell calculation. The second software component, GAMBET, is a new Monte Carlo code for radiation transport that incorporates effects of the complex electric and magnetic fields at the radiation target. The ray-tracing approach exhibits good convergence in calculations for the diode geometry of the compact radiography (CRAD) program at Lawrence Livermore National Laboratory. With a $1.5 \mathrm{MV}, 30 \mathrm{~ns}$ driver, we predict that the diode can produce a beam with axial length $\sim 1 \mathrm{~mm}$ that generates isotropic bremsstrahlung radiation exceeding $1 \mathrm{rad}$ at $1 \mathrm{~m}$. The ray-tracing procedure encounters convergence problems when applied to the rod-pinch geometry, a configuration used in several pulsed radiographic machines. We observe a fundamental difference in the nature of electron orbits in the two diodes. There is an increased chance for particle-orbit feedback in the rod pinch, so that equilibrium solutions are sensitive to small changes in emission characteristics.
\end{abstract}

DOI: 10.1103/PhysRevSTAB.9.020401

PACS numbers: 52.59. $-\mathrm{f}$

\section{INTRODUCTION}

Flash x-ray radiography using pulsed-electron accelerators is a key diagnostic in the investigation and understanding of explosively-driven objects [1,2]. The radiographic source must have a small spot size and short duration to provide adequate spatial and temporal resolution. The conditions are typically achieved by focusing an intense electron beam on a high- $Z$ bremsstrahlung target. Two types of tools have been developed to meet these requirements: linear induction accelerators and pulsedpower drivers. Induction accelerators (such as DARHT [3] and FXR [4]) generate high-energy, high-current, short-pulse beams ( $\sim 20 \mathrm{MeV}, \sim 2 \mathrm{kA}, \sim 50 \mathrm{~ns})$ that can be focused by conventional magnetic lenses to a small spot. These beams produce a hard spectrum of bremsstrahlung photons predominantly in the forward direction. Therefore, the axial length of the radiation spot (determined by the electron range in the target material) is not critical to the application. For a softer radiation spectrum, less-costly pulsed electrostatic accelerators are used to drive pinched-beam diodes in the voltage range from hundreds of $\mathrm{kV}$ to a few MV [5-7]. In these devices, there is no external focusing system. If the current is high enough, the magnetic field generated by the beam itself confines electrons to a small spot. In the compact radiography (CRAD) program our goal is to operate a small pinched-beam diode (Fig. 1(a)) in close proximity to the test object. In this application the radiation distribution should be nearly isotropic; therefore, the axial length of the radiation spot must be small to achieve good spatial resolution.
This paper describes simulation tools that can model self-consistent electron beam dynamics and radiation generation as they relate to the CRAD program. The coupled radiographic software suite includes two- and threedimensional conformal mesh generators, finite-element codes to determine applied electric and magnetic fields, an orbit-tracking program that includes self-consistent Child-law emission and beam-generated magnetic fields, and an electron/photon Monte Carlo code that incorporates the effects of fields on backscattered electron orbits. For the beam-dynamics calculation, we apply ray-tracing techniques [8-15] instead of the particle-in-cell methods often used for radiographic simulations [16]. Quasistatic calculations are sufficient for pinched-beam diodes because the transit times of electrons and ions are short compared to typical pulse lengths. Ray tracing is a more efficient procedure than the particle-in-cell method because it circumvents the need to calculate a complete rise-time history. TRAK (the beam-dynamics component of the radiographic suite) has advanced capabilities to address super-pinchedelectron beams. The code accurately determines selfconsistent magnetic fields generated by both the beam and current flow on internal electrodes. We used the new GAMBET code to determine the properties of emitted bremsstrahlung radiation. GAMBET is unique among Monte Carlo codes in its use of two- or three-dimensional conformal meshes to define system geometry and to record dose distributions. The program has three features that are critical to the application: (1) it accepts primary-particle input directly from TRAK, (2) it can include the effect of 
a)

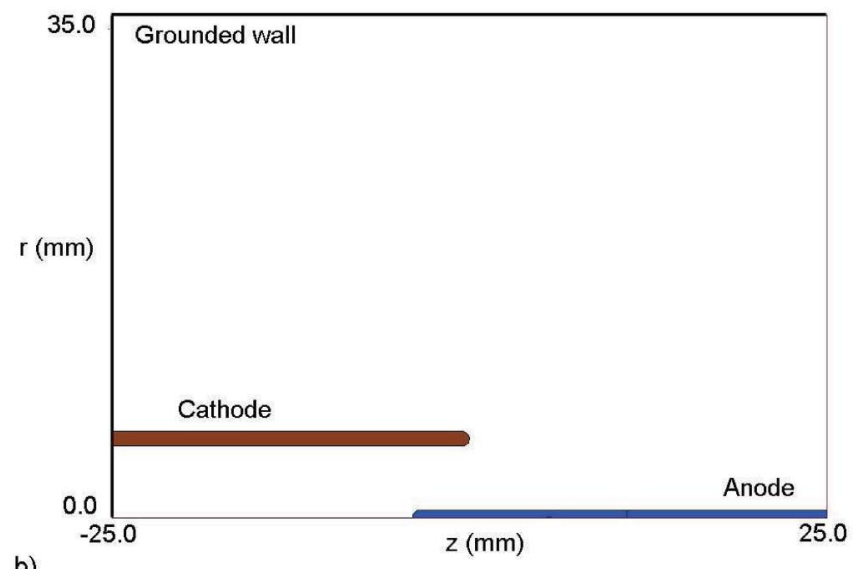

b)

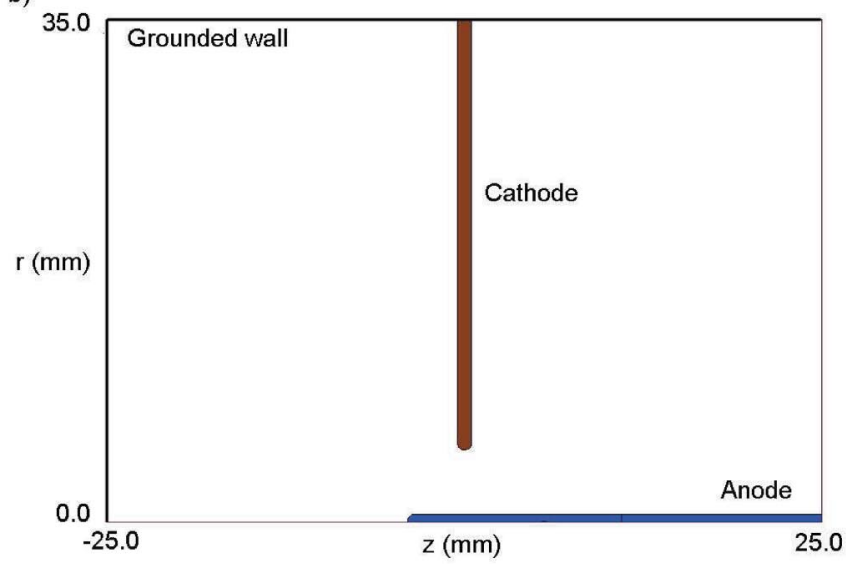

FIG. 1. (Color) Pinched-beam diode geometries for pulsedbeam radiography. The $z-r$ plot shows dimensions in $\mathrm{mm}$ for the calculations of this paper. (a) CRAD diode. (b) Rod-pinch diode.

complex calculated electric and magnetic fields on backscattered electrons, and (3) it can accurately model material objects of any shape.

The following section describes the features of TRAK that impact pinched-electron beam calculations. Section II summarizes simulations of the CRAD diode [Fig. 1(a)]. The investigation centers on next-generation experiments using a $1.5 \mathrm{MV}, 30 \mathrm{~ns}$ pulsed-power driver. The wellconverged solutions derived through the ray-tracing method show good pinches with net current $\sim 30 \mathrm{kA}$ where the axial length for electron deposition on the target is $\sim 1 \mathrm{~mm}$. In contrast, the ray-tracing method gives poor results for the rod-pinch diode [Fig. 1(b)], a geometry used in many radiographic machines [5,17]. Although the average total current is close to reported values, the calculations exhibit large oscillations between iteration cycles. The results give insight into differences between the CRAD and rod-pinch diodes. Section IV discusses unique features of the GAMBET code for modeling electron/photon transport in the pinched-beam environment. Finally, Sec. V presents GAMBET results for generation of bremsstrahlung radiation by the CRAD diode, including the radiation spot size and the angular variation of dose.

\section{TRAK FEATURES FOR PINCHED-ELECTRON BEAMS}

All ray-tracing codes use the same method to find electric fields with space-charge effects in nonrelativistic steady-state electron guns [18]. The beam is represented by a finite number $N$ of model particles that carry a fraction $I_{n}$ of the total beam current and move along single-particle streamlines. Starting from a solution for vacuum electric fields, the codes calculate model electron orbits by advancing in small time steps $\Delta t$. At each step, a charge $I_{n} \Delta t$ is deposited at the present location on the computational mesh. The electrical field is updated to include the space charge, and then the orbits are recalculated in the modified field. If electrons are emitted from a Child-law surface, the total beam current may vary with the evolving local electric field. The process is repeated over several cycles. With sufficient space-charge averaging, the procedure converges to a self-consistent electric-field solution. The main differences between codes are (1) the type of mesh employed and (2) the method for the electric-field calculation.

There are significant differences between ray-tracing programs in calculations of the self-consistent magnetic fields of relativistic beams. The method of internal ray counting used in early codes $[8,9]$ cannot handle the highly nonlaminar electron motion in pinched beams. The use of a simple mesh to record included beam current and to determine $B_{\theta}(r, z)$ can resolve the problem of complex electron motion, but there are two issues to address:

(i) Small differences in the field calculation give large errors in the net force for relativistic beams where the electric and magnetic forces closely balance.

(ii) The anode and parts of the cathode in the diodes of Fig. 1 are internal to the electron flow. Therefore, surface currents on electrodes must be added to the ion and electron current to find the total toroidal field.

Figure 2 shows the method used in TRAK [19] to address the problems. The program uses the same conformal mesh to calculate electric and magnetic fields. The rationale is that the fixed-potential electrode surfaces also define the paths of pulsed-current flow. The primary task in the magnetic-field calculation for a cylindrical system is to find the total current carried by electrons, ions and electrodes enclosed within each mesh node, $I_{\text {inc }}(r, z)$. Values of toroidal magnetic field at the nodes can then be determined from the equation

$$
B_{\theta}(r, z)=\frac{\mu_{0} I_{\text {inc }}(r, z)}{2 \pi r} .
$$

TRAK accumulates current on element facets (Fig. 2) rather than current density in element volumes. If a particle carrying current $I_{n}$ passes through a facet in a positive sense with respect to $z$, then the facet current is augmented 


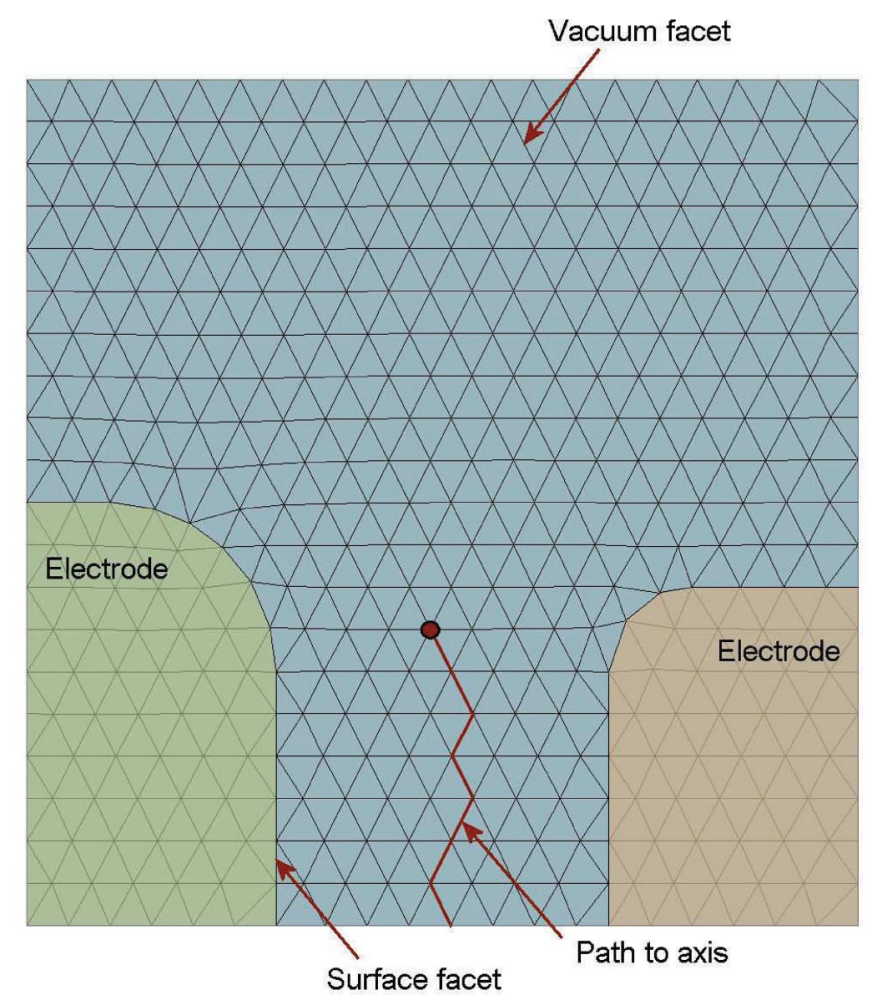

FIG. 2. (Color) Calculation of beam-generated magnetic fields on a conformal triangular mesh. Beam current is recorded on element facets. Current included within a vacuum node is the sum of facet currents on a path to the axis.

by $I_{n}$. Because all current that enters a vacuum element must leave the element, the facet assignment automatically satisfies the condition $\nabla \cdot \mathbf{j}=0$. When orbit tracking has been completed, we can find $I_{\text {inc }}$ at a node by summing current contributions along a path of connected facets to the axis (Fig. 2). The value is independent of the choice as long as the path does not pass through an electrode. The method correctly calculates contributions of counterstreaming electrons and ions within the beam-transport volume.

We must make special provisions to include contributions from current flow along electrodes. The approach in TRAK is to assign particle current to the surface facets of the source and target electrodes. The sign of the current depends on the flow direction along the electrode. For the positive flow direction, the source is connected to the lefthand boundary and the target is connected to the right-hand boundary. The method of treating Child-law emission in TRAK [20] helps in the assignment of source current. All ray-tracing codes handle space-charge-limited emission by initiating particles from a special surface near the source. TRAK assigns space charge in the volume between the source and emission surfaces by initially backtracking particles at constant velocity from the emission surface until they intersect a source facet. For a particle with positive flow direction, the code assigns a current $+I_{n}$ to the facet. The program then tracks particles forward from the emission surface until they intersect a target facet. In this case, the facet current is increased by $+I_{n}$.

After completing orbit tracking and assignment of currents to vacuum and surface facets, TRAK uses the following procedure to find $I_{\text {inc }}$ at nodes:

(1) Set $I_{\text {inc }}$ equal to zero at all on-axis nodes.

(2) Find the intersection points of electrode surfaces with the axis. Follow the path outward, keeping a running sum of facet currents. Assign the summed current to the nodes of the surface. This procedure requires that an electrode surface consists of a contiguous set of facets that intersects the axis at only one point.

(3) Assign current to nodes in the vacuum region by working outward from the axis in radial layers. At each node, seek a facet that connects inward to an electrode surface or a vacuum node with an assigned value of $I_{\text {inc }}$. Set the included current equal to the sum of $I_{\text {inc }}$ and the facet current.

The method ensures a good balance of accuracy between electric and magnetic-field calculations. Furthermore, the resulting quantity $B_{\theta}$ includes contributions from both positive and negative particles as well as electrode currents. Particle contributions are correct even if they follow reflex orbits.

\section{RADIOGRAPHIC DIODE SIMULATION RESULTS}

The primary goal of the work described in this paper was to determine CRAD diode performance with a secondgeneration pulsed-power driver (1.5 MV, $30 \mathrm{~ns}$ ). Previous calculations of electron current and radiation generation for the present CRAD experiment at 1.2 MV [21] were consistent with observed results to within the accuracy limits of the diode voltage and current measurements. Figure 1(a) shows the geometry of the CRAD diode. It consists of a tungsten rod anode $1 \mathrm{~mm}$ in diameter inside a thin tubular aluminum cathode with inner radius $R_{c}$. The cathode and anode overlap a distance $L_{a c}$. With fixed anode diameter, the only two variable parameters are $L_{a c}$ and $R_{c}$. In operation the diode is located at the end of a transmission line with applied axial magnetic-field insulation $\left(B_{z}\right)$. We have confirmed with TRAK simulations that the axial field at the diode is quite small and that electron loss in the transition region from axial to toroidal field insulation is moderate. The magnetically-insulated transmission line has an impedance of $50 \Omega$. Therefore, the ideal matched diode should draw a total current of $30 \mathrm{kA}$. In applications to close-proximity radiography, we need a radiation spot size that does not exceed $1 \mathrm{~mm}$ in any direction. The size is automatically constrained in the radial direction by the anode diameter. Confinement in the axial direction must be performed by the self-pinching process. Here, the intense toroidal field created by the diode current pushes electrons to the tip of the anode. 
An electron pinch can be achieved only if there are counter-streaming ions $[20,22]$. Because of the short voltage pulse length, we can employ a relatively small cathode-anode gap $(\sim 4.5 \mathrm{~mm})$ in the CRAD diode without significant effects of diode closure by expanding plasmas. Consequently, the cathode and anode are subject to high electric fields ( $\geq 100 \mathrm{MV} / \mathrm{m}$ on the cathode and $\geq$ $1000 \mathrm{MV} / \mathrm{m}$ on the anode). At these levels we expect rapid explosive plasma emission providing an unlimited source of electrons and ions. In the experiments we normally apply a thin hydrogenous coating to the anode surface to ensure rapid ion generation. In the simulations, our choice of emission surfaces for electrons and ions depended on $L_{a c}$. The ion surface always began at the anode tip and extended a few millimeters past the last point where the ion trajectories intersected the cathode. Ion trajectories that did not contribute space charge to the cathode emission region had little effect on the net diode current. The electron emission surface started on the inside of the cathode several millimeters to the left of the anode tip. In a typical run, the steady-state electric field was repulsive in this area so that first set of model electrons did not contribute to the diode current. The electron emission surface continued around the cathode rim and extended about $1.5 \mathrm{~mm}$ along the outer surface. Beyond this the surface electric field was greatly reduced so that model electrons would carry negligible current. We assumed protons as the ion species. Because ions suffered only a small deflection in the toroidal magnetic field, the choice of species had a negligible effect on the steady-state solution.

We defined a variable-resolution mesh with small elements $(\sim 0.1 \mathrm{~mm})$ in the region surrounding the cathodeanode overlap. TRAK automatically picked appropriate orbit integration time steps for the ions and electrons. We set up the run to generate five model electrons per emission facet and four ions. The baseline run included 430 model electrons and 252 model ions. The strong magnetic-field effects and complex electron orbits presented a challenge to the code. For good convergence it was necessary to correct the space charge and beam current gradually and extend the run to a large number of iteration cycles $(\sim 70)$. Averaging of charge and current was controlled by the parameter $\epsilon$. The space-charge density after orbit recalculation was equal to $\epsilon$ times the new value plus $(1-\epsilon)$ times the averaged value from previous cycles. In the simulations, the choice $\epsilon=0.05$ over the final 50 cycles gave good convergence.

We used parameters $R_{c}=5.0 \mathrm{~mm}$ and $L_{a c}=4.0 \mathrm{~mm}$ for the baseline run. The blue curve in Fig. 3 shows the variation of total diode current with cycle number. The initially high value dropped as the space-charge density grew slowly through the averaging process. The late-time current approached $29.3 \mathrm{kA}$, with less than $1 \%$ variation between cycles. The run time on $3.2 \mathrm{GHz} 32$-bit computer was $9.5 \mathrm{~min}$.

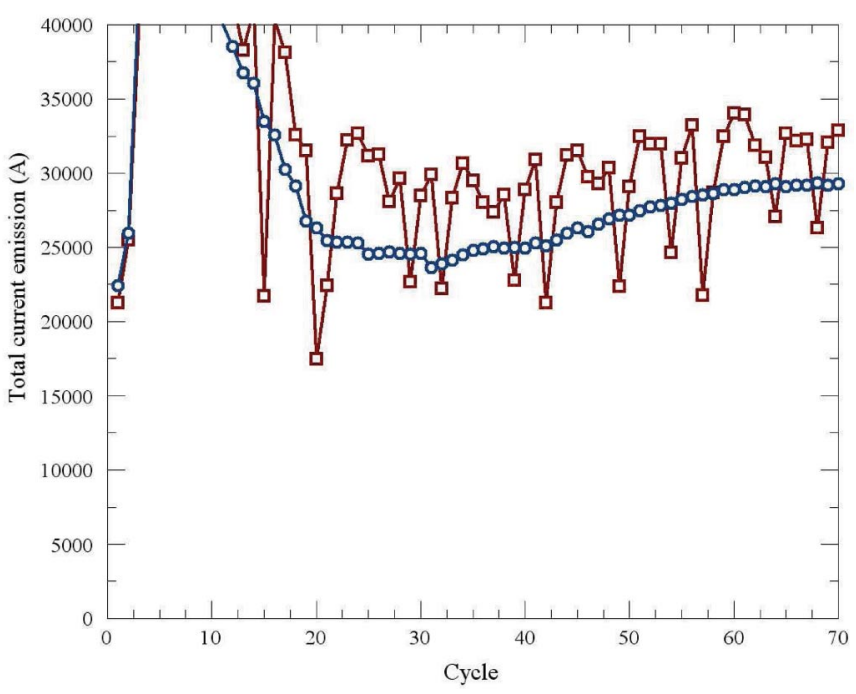

FIG. 3. (Color) Total simulation current as a function of iteration cycle with a charge averaging factor of $\epsilon=0.05$. Blue line: CRAD diode. Red line: rod-pinch diode.

Figure 4 show plots of selected ion and electron orbits along with calculated lines of constant electrostatic potential. The toroidal magnetic field had little effect on the ions [Fig. 4(a)], which moved outward along smooth curves determined by the electric-field distribution. For the choice of emission surface, the ions illuminated all inner portions of the cathode. The resulting positive charge strongly influenced the space-charge-limited electron current density. The toroidal magnetic field dominated electron motion [Fig. 4(b)]. With $30 \mathrm{kA}$ of current flow, electrons emitted near the cathode rim were swept to the anode tip. In the baseline run, $75 \%$ of the electrons struck the anode within $0.8 \mathrm{~mm}$ of the tip (the length for $95 \%$ collection was $1.4 \mathrm{~mm}$ ). In the plot of Fig. 4(b) there appears to be considerable electron activity to the left of the anode tip. These electrons actually moved away from the anode under the influence of the negative space charge of the pinched beam. They followed complex reflex orbits in the dead space to the left of the pinch. Figure 5(a) clarifies the nature of electron flow. Here, the orbits are plotted in a gray scale with darkness proportional to the model-particle current. In this plot the reflexing electrons almost disappear because they carry very little current. The dark trajectories show that the bulk of the electron current flows in a smooth stream from the upstream edge of the cathode to the anode tip.

Although the ions made only a small contribution to the total diode current, their space charge had a strong effect on the space-charge-limited electron flux. To illustrate the effect of ions, we deactivated ion emission from the anode. For monopolar flow, the total current dropped to $11.6 \mathrm{kA}$. Figure 5(b) shows the resulting electron orbits. At the reduced current, electron motion was no longer dominated by the toroidal magnetic field. The collection region for 

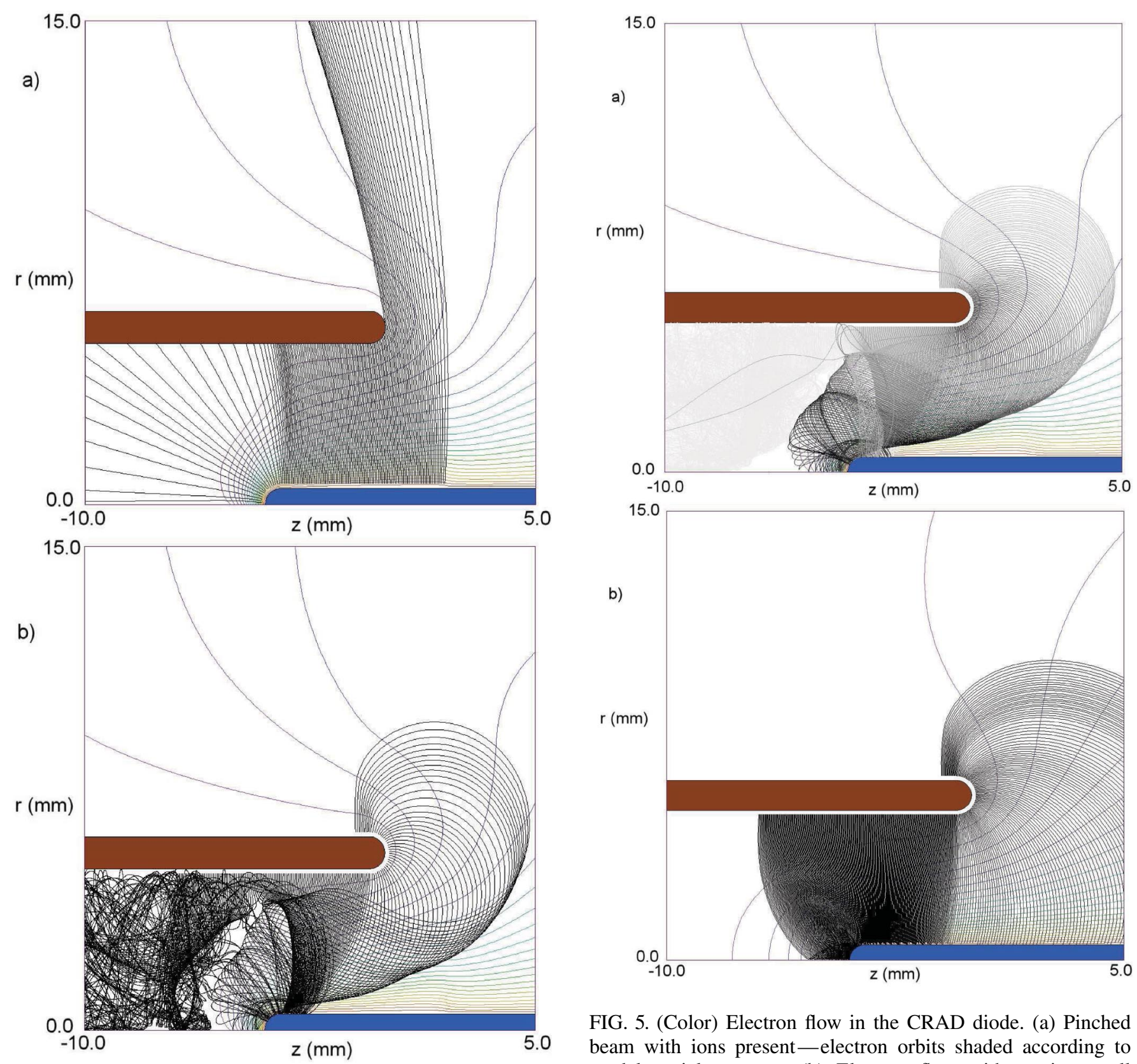

FIG. 5. (Color) Electron flow in the CRAD diode. (a) Pinched beam with ions present-electron orbits shaded according to model-particle current. (b) Electron flow with no ions-all orbits plotted.

FIG. 4. (Color) Calculated trajectories and equipotential lines for the CRAD diode with $R_{c}=5.0 \mathrm{~mm}$ and $L_{a c}=4.0 \mathrm{~mm}$. (a) Ions. (b) Electrons

electrons on the target exceeded $12 \mathrm{~mm}$ in length. In the absence of the high negative space charge of a pinched beam, electrons from the region to the left of the anode tip were able to leave the cathode and travel directly to the anode.

We performed a series of runs varying $L_{a c}$ at fixed cathode inner radius $R_{c}=5.0 \mathrm{~mm}$. Figure 6 shows the variation of electron current and distance from the anode tip for collection of $75 \%$ of the electrons. Clearly, some electrode overlap was required to generate sufficient current to maintain the pinch. There was little change in the

electron current in the range $L_{a c}=4.0 \mathrm{~mm}$ to $9.0 \mathrm{~mm}-$ the electron flow was determined mainly by the pinch dynamics rather than the effective diode area. There was an increase in the ion loss current with overlap, so large values of $L_{a c}$ are undesirable. The choice $L_{a c} \sim R_{c}$ provides a good pinch and gives a total diode current that is matched to a $50 \Omega$ transmission line.

For some choices of $L_{a c}$, a fraction of the electrons from the cathode rim skimmed the anode before reaching the tip. The TRAK code stopped the electrons at this point, accounting for the enhanced values of the $75 \%$ collection length in Fig. 6(b). The resulting average value of axial collection length in the range $4.0 \mathrm{~mm} \leq L_{a c} \leq 9.0 \mathrm{~mm}$ was $1.2 \mathrm{~mm}$. 

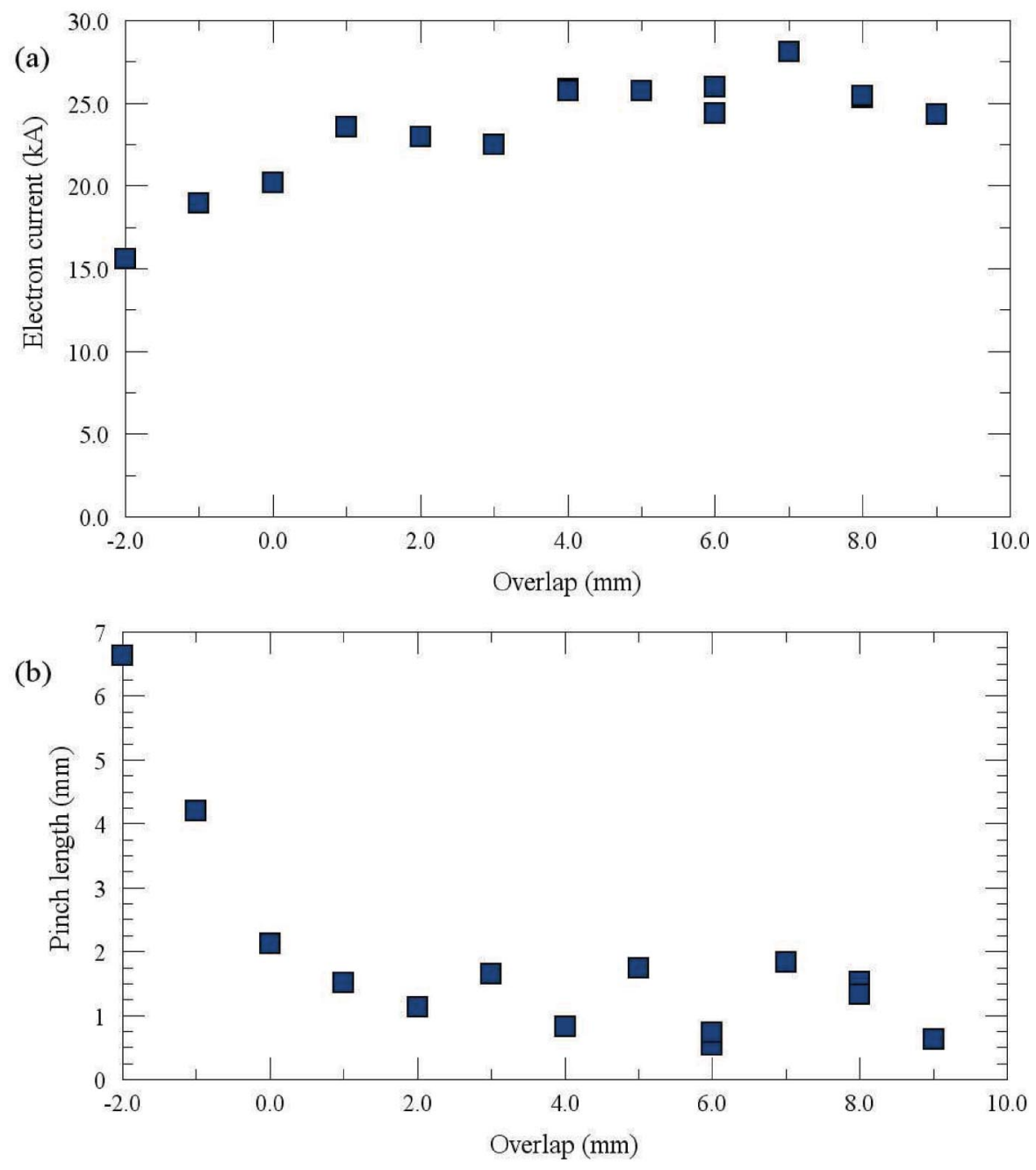

FIG. 6. (Color) CRAD diode performance as a function of cathode-anode overlap $L_{a c}$ with a fixed cathode inner radius $R_{c}=5.0$ mm. (a) Electron current. (b) Axial distance from anode tip for collection of $75 \%$ of the electron current.

There are two reasons why the energy deposition length in a physical system may be lower: (1) there is a high probability for reemission of electron striking the anode at grazing incidence and (2) the electrostatic radius of the anode may be larger than the physical radius because of surface plasma expansion.

In a second set of runs, we used the fixed overlap value $L_{a c}=4.0 \mathrm{~mm}$ and varied $R_{c}$. The results are listed in Table I. The total current decreased and the electron deposition length increased at larger radii. The relative change

TABLE I. CRAD diode performance as a function of cathode inner radius.

\begin{tabular}{lcccc}
\hline \hline$R_{c}(\mathrm{~mm})$ & $I_{\text {tot }}(\mathrm{kA})$ & $I_{\text {elec }}(\mathrm{kA})$ & $I_{\text {ion }}(\mathrm{kA})$ & $\mathrm{Z75}(\mathrm{mm})$ \\
\hline 5.0 & 29.31 & 25.87 & 3.44 & 0.83 \\
6.0 & 27.44 & 24.27 & 3.18 & 1.20 \\
7.0 & 25.10 & 22.36 & 2.74 & 1.13 \\
8.0 & 22.53 & 20.17 & 2.37 & 1.50 \\
\hline \hline
\end{tabular}

in current was far less than would occur for laminar spacecharge-limited flow in a cylindrical diode. Again, the diode performance was dominated by the pinched-beam dynamics.

We also performed calculations for a generic rod-pinch geometry [Fig. 1(b)]. Again, the anode was a $1 \mathrm{~mm}$ diameter tungsten rod that overlapped the cathode. The only difference was the shape of the cathode, a plate with an aperture. For a comparison with the baseline CRAD diode, we picked a $5.0 \mathrm{~mm}$ aperture radius and an anode/cathode overlap of $4.0 \mathrm{~mm}$. Although the average properties of the resulting pinched-electron flow were similar to that in the CRAD diode, there was a major difference in convergence characteristics for the ray-tracing solution. The red curve in Fig. 3 plots the calculated total current for the rod pinch as a function of cycle number. Again, we used strong charge and current averaging $(\epsilon=0.05)$. Although the curve had the same general variation, there were large intercycle oscillations which persisted even with very strong damping $(\epsilon=0.025)$. The code behavior for the rod pinch was not 
specific to our choice of geometry. We have also used TRAK to model the Cygnus diode at Los Alamos National Laboratory [7,23]. Although the average current at 2.25 MV was near the reported value of $60 \mathrm{kA}$, we observed strong current variations between cycles.

To get insight into the nature of the problem, we can compare results for the generic diode calculation on two cycles near the end of run. Figure 3 shows that the current had a high value of $32.9 \mathrm{kA}$ on cycle 70 and a low value of $26.3 \mathrm{kA}$ on cycle 68 . Comparing the two solutions, the total current varied about $\pm 10 \%$ about an average of $29.6 \mathrm{kA}$. The electron current varied $\pm 11.5 \%$ while the change in ion current was only $\pm 3.5 \%$. The axial length for collection of $75 \%$ of the electrons was $1.1 \mathrm{~mm}$ in both cases. The numbers imply that the intercycle variations resulted mainly from changes in the electron flow characteristics. Figure 7 shows plots of model electron orbits on cycles 68 and 70 . There was little difference in the orbits of electrons emitted from the front face of the cathode (right-hand side). These orbits were similar to those in the CRAD diode [Fig. 4(b)]. On the other hand, there were substantial differences in the equipotential distribution and the nature of electron orbits on the downstream face. In the CRAD diode, all electrons created on the emission surface either followed relatively direct streamlines to the anode or were suppressed by the pinched-beam space charge. Therefore, there was no feedback mechanism where electrons with significant current could return to a region of strong emission on the cathode. In contrast, there were complex reflex orbits on the downstream side of the cathode in the rodpinch diode. Groups of reflex electrons followed paths that returned them to the main flow volume. Therefore, strong emission on one cycle could suppress electron generation on a subsequent cycle, even with strong charge averaging. We cannot conclude that the results of Fig. 7 imply an instability of the rod-pinch diode because the ray-tracing code does not replicate time-dependent physical processes. Nonetheless, we can state that electron orbits in the rodpinch diode are more complex than those of the CRAD diode, and that the process of space-charge-limited emission is more sensitive to small changes in the electron flow pattern.

\section{GAMBET CAPABILITIES FOR PINCHED- ELECTRON BEAMS}

GAMBET is a new Monte Carlo code for electron/photon/ positron transport in matter. In this section we discuss some program features that are useful for pinched-beam radiography calculations. GAMBET combines technology we have developed for finite-element calculations with the PENELOPE radiation-physics package [24]. PENELOPE performs the Monte Carlo tasks such as generation of atomic cross sections, prediction of single-particle interactions with matter, creation of secondary particles, and determination of the effects of collisions. Our package handles run
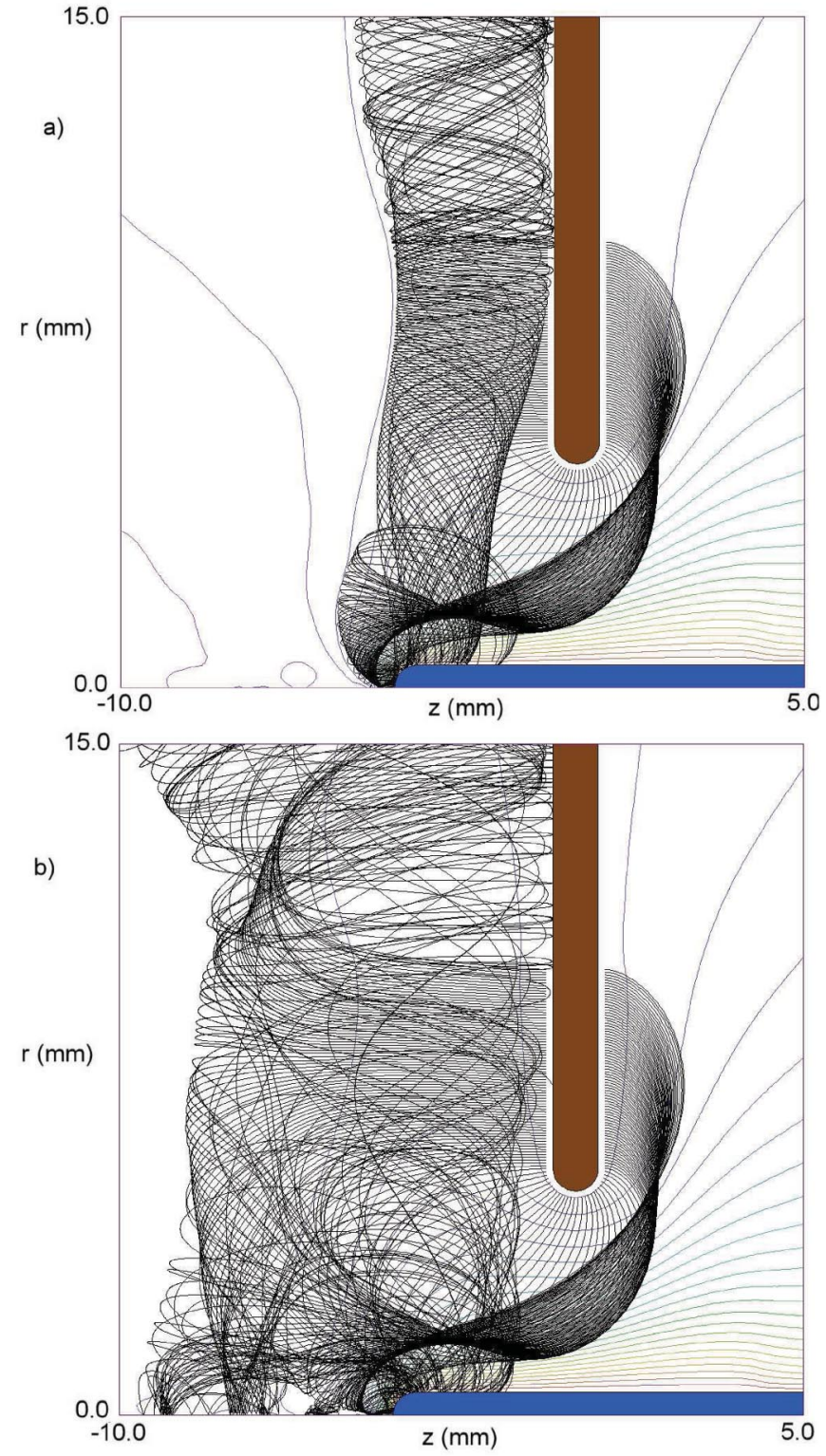

FIG. 7. (Color) Electron flow in the generic rod-pinch diode. (a) Cycle 68. (b) Cycle 70.

control, energy scoring, and all matters related to the division of space. Tasks include the organization of input and output data for large distributions of particles, calculation of statistics, identification of material boundaries, generation of escape-particle records, variance reduction techniques, and records of spatial variations of dose.

GAMBET has three unique features compared to previous Monte Carlo codes [24-28]:

(i) It employs conformal two- and three-dimensional meshes of volume elements to describe geometries.

(ii) It is part of a unitized package with components to compute electric and magnetic fields and self-consistent input particle distributions.

(iii) It includes interactive graphical utilities to prepare input and to analyze results. 
With the conformal mesh approach, there is no difficulty treating intricate assemblies and boundaries of arbitrary shape. Furthermore, the elements can be used as subzones for energy scoring. We can therefore employ postprocessor features developed for finite-element analysis to create dose plots and to perform integrals. The main drawback is that some effort is required to create a mesh. The issue is mitigated by the factors: (1) the mesh generation process is largely automatic and (2) in most problems the user would have already created a mesh to model fields and to generate input particle distributions.

All GAMBET runs require a geometry mesh which describes boundaries for particle trajectories and the distribution of materials and voids in space (Fig. 8). The geometry mesh may be either two dimensional (cylindrical or planar symmetry) or three dimensional. The key to practical program operation is the use of structured conformal meshes. Although elements may have different shapes, their relationship to one another follows ideal triangular or cubic logic. This feature enables fast element searches and boundary identification. The program can also load independent meshes for electric fields or magnetic fields calculated with other components of the radiographic suite. GAMBET accepts direct input of electron or positron distributions from the TRAK and OMNITRAK codes. Similarly, the particle output files can be used as input for subsequent GAMBET runs or ported to the orbit-tracking programs.

A GAMBET run consists of the following steps:

(1) Load and check the geometry mesh. Optionally, load meshes and node values for field calculations.

(2) Load source particles (electrons, photons, or positrons). The program reads primary-particle parameters from a text list. The list could be prepared by the TRAK and OMNITRAK programs, a previous GAMBET run or a user program. The software suite includes GENDIST, a utility to create standardized lists from a set of source parameters.

(3) Load the material identities of regions of the geometry mesh and pass them to the PENELOPE routines for the calculation of cross sections.

(4) Follow a specified number of showers for each primary particle. The program can operate in the standard Monte Carlo mode, recording energy deposited in elements per primary particle (eV/primary). The program can also associate flux or current with each primary particle. In this case, GAMBET determines deposited dose rate for incident particle or photon beams.

(5) Finally, GAMBET creates output files and records results of statistical calculations in a listing file. The program also creates an escape file, a list of parameters for particles that leave the solution volume. The dose file contains a record of deposited energy in each element of the geometry mesh. The contents of this file can be plotted or viewed with GBVIEW, an interactive postprocessor.

\section{GAMBET SIMULATIONS OF THE CRAD DIODE}

We used GAMBET to determine the characteristics of bremsstrahlung radiation generated by the CRAD baseline diode $\left(R_{c}=5.0 \mathrm{~mm}, L_{a c}=4.0 \mathrm{~mm}\right)$. The simulations included motion of backscattered electrons in the intense electric and magnetic fields near the target, a task that would be difficult or impossible with other Monte Carlo codes. The calculations use three outputs from the TRAK simulations described in Sec. III: (1) the distribution of electrons incident on the target, (2) the electric-field distribution near the target resulting from combined effects of applied voltage and electron and ion space charge and (3)

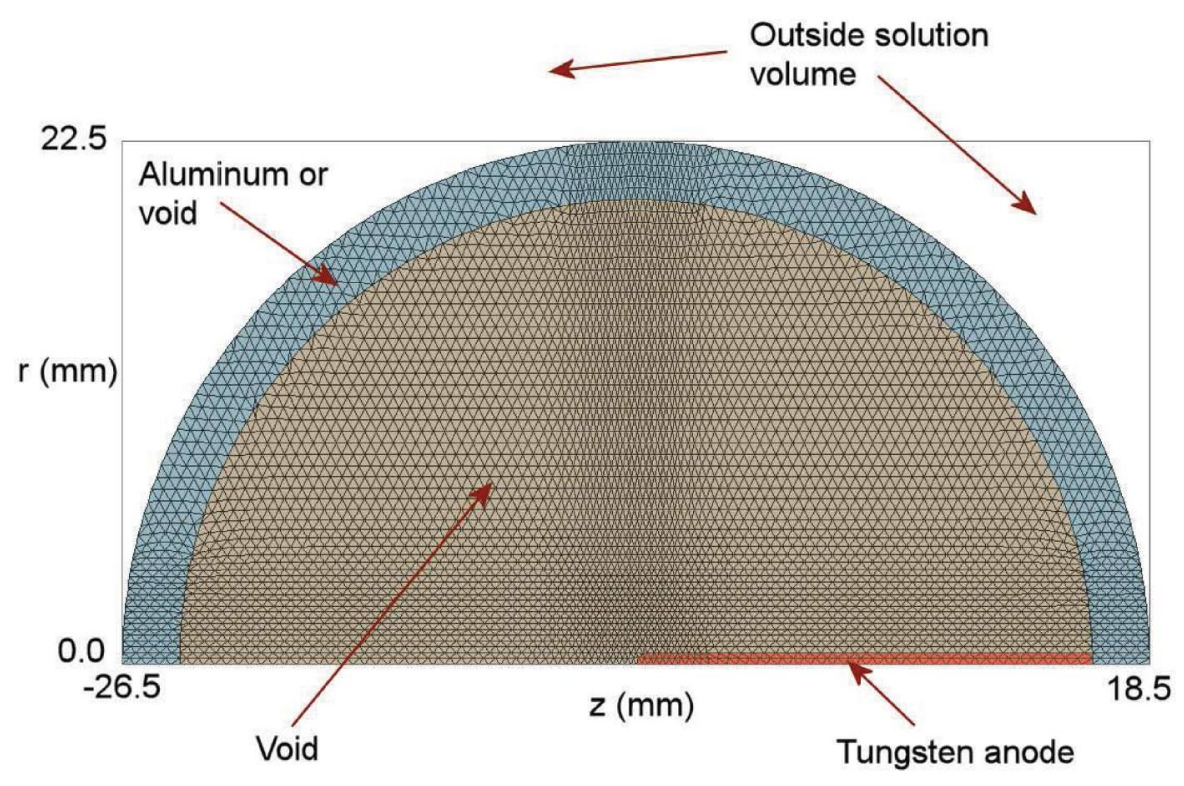

FIG. 8. (Color) GAMBET geometry mesh for the CRAD baseline simulation. 
the toroidal magnetic-field distribution resulting from electron and ion flow as well as current along the anode and cathode surfaces.

The first step to prepare the run was to filter the particle file created by TRAK with the GENDIST utility. We removed ions and the small fraction of model electrons that did not reach the anode. Besides position and momentum, the TRAK particle file contains the model-particle currents. GAMBET can use this information to find bremsstrahlung dose rates in surrounding materials. Incident electrons scatter and lose energy in the tungsten anode. Backscattered electrons with reduced energy are confined near the anode by the electric field and the intense toroidal magnetic field ( $\sim 12 \mathrm{~T}$ on the anode surface). Therefore, the GAMBET geometry mesh need not cover the same volume as the electric mesh for the TRAK solution [Fig. 1(a)]. For convenience, we chose a spherical solution volume of radius $R_{s}=22.5 \mathrm{~mm}$ centered on the anode tip (Fig. 8). There were four regions in the solution volume. The region outside the sphere was an external void. Particles that left the solution volume were added to the escape file. The final position and momentum were calculated by interpolation to the curved boundary of the solution volume. The anode had the material properties of tungsten, with zero internal electric and magnetic fields. The adjacent space had void properties. Here there were no material interactions. Photons traveled in ballistic orbits while electrons and positrons moved under influence of the electric and magnetic fields. The third region was a spherical shell that was assigned different properties depending on the purpose of analysis:

(i) With a void assignment to the shell, the escape file gave the available bremsstrahlung photon distribution without the effects of intervening materials. After filtering the escape file to remove a small component of positrons, we could determine the energy spectrum, angular distribution and total photon yield. We could also back-project the escaping photon trajectories by a distance $-R_{s}$ to find the effective size of radiation spot.

(ii) With the assignment of the material characteristics of aluminum to the shell, we could determine the angular dependence of bremsstrahlung dose at an average radius of $21.25 \mathrm{~mm}$. This figure could be extrapolated to other distances using a $1 / r^{2}$ variation.

(iii) We could assign different material identities and densities to determine the effects of a vacuum chamber wall or other obstruction on the bremsstrahlung distribution.

The particle input file for the calculations included 400 primary electrons. We used a shower multiplication factor (showers/primary) of 60 for a total of 24000 showers. To improve statistics, we included a forcing factor of 100 for bremsstrahlung interactions. The run time was about $29 \mathrm{~min}$. With the outer shell set to the void condition, the escape file contained 607489 photons with an average energy of $278.0 \mathrm{keV}$. Assuming that each primary electron had a kinetic energy of $1.5 \mathrm{MeV}$, the total radiation yield was

$$
Y=\frac{607489 \times 278}{60 \times 100 \times 400 \times 1500}=0.047 .
$$

An important quantity for this application is the effective axial length for photon generation at the target. The GAMBET postprocessor can perform several types of back projection. In this case, we projected photon orbits recorded on the outer sphere by a distance $-22.5 \mathrm{~mm}$ and called for a calculation of a histogram of photon flux as a function of the modified axial location. Figure 9 shows the probability density function $f(z)$ and the cumulative distribution function $F(z)=\int f(z) d z$. Over $85 \%$ of the photons where produced within $1 \mathrm{~mm}$ of the anode tip, a result consistent with the TRAK results for electron deposition.

Figure 10 shows dose-rate distribution contours in the anode along with selected primary electron orbits. The orbits included effects of energy loss and scattering. Backscattered and transmitted electrons that reentered the void moved under the influence of the electric and magnetic fields calculated by TRAK. Electrons that suffered multiple backscattering events followed drift orbits in the $+z$ direction, opposite to the motion of incident electrons in the pinch. The drifts had only a small effect on the dose distribution in the anode and the radiation spot because the electrons lost most of their energy near the tip.

We can calculate the bremsstrahlung photon dose available for radiography by assigning the material properties of aluminum to the outer shell. Except for a small number of positrons, the strong electric and magnetic fields ensured that only photons reached the shell. Therefore, the recorded energy deposition gave a good approximation for

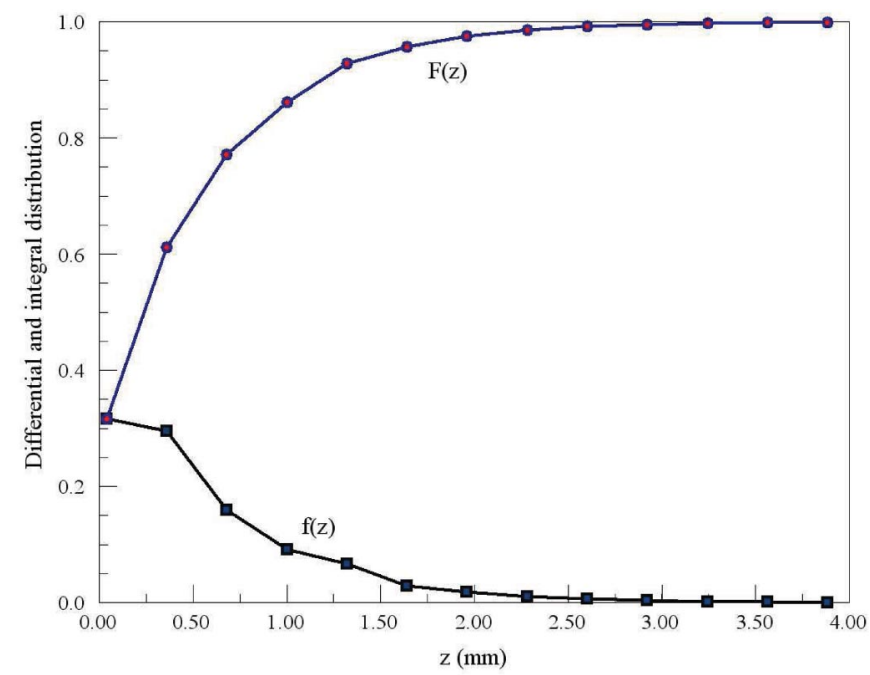

FIG. 9. (Color) Radiation spot size for the CRAD baseline simulation, determined by back-projecting photon trajectories. Photon probability density function $f(z)$ and cumulative distribution function $F(z)$ as a function of distance from the anode tip. 


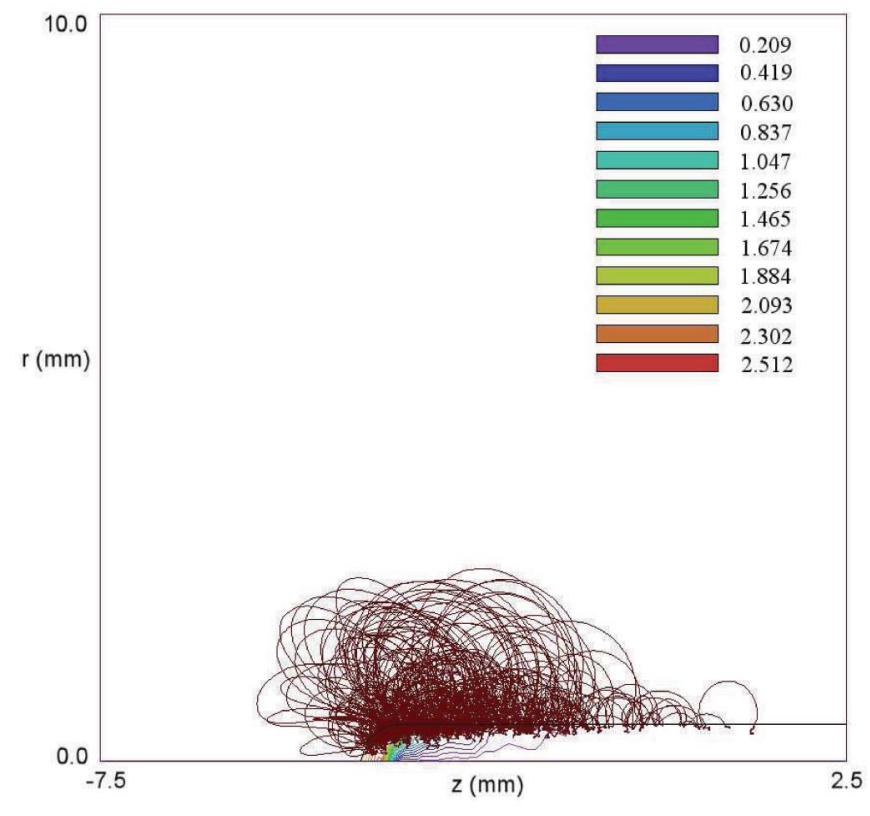

FIG. 10. (Color) Selected primary electron orbits and dose-rate distribution $(\mathrm{Gy} / \mathrm{s})$ in the target for the CRAD baseline simulation. Multiply values by $10^{15}$.

the photon dose. The elements of the shell had a transverse width of about $0.5 \mathrm{~mm}$. We can therefore infer spatial variations of dose on the order of the element size, or we can introduce energy-conserving smoothing to reduce statistic variations at the expense of reduced resolution. Figure 11 shows the smoothed dose distribution in the shell. There was good uniformity over the full solid angle with some reduction in the forward and backward directions because of target absorption. The hot spot marked $a$ resulted from a small number of backscattered electrons

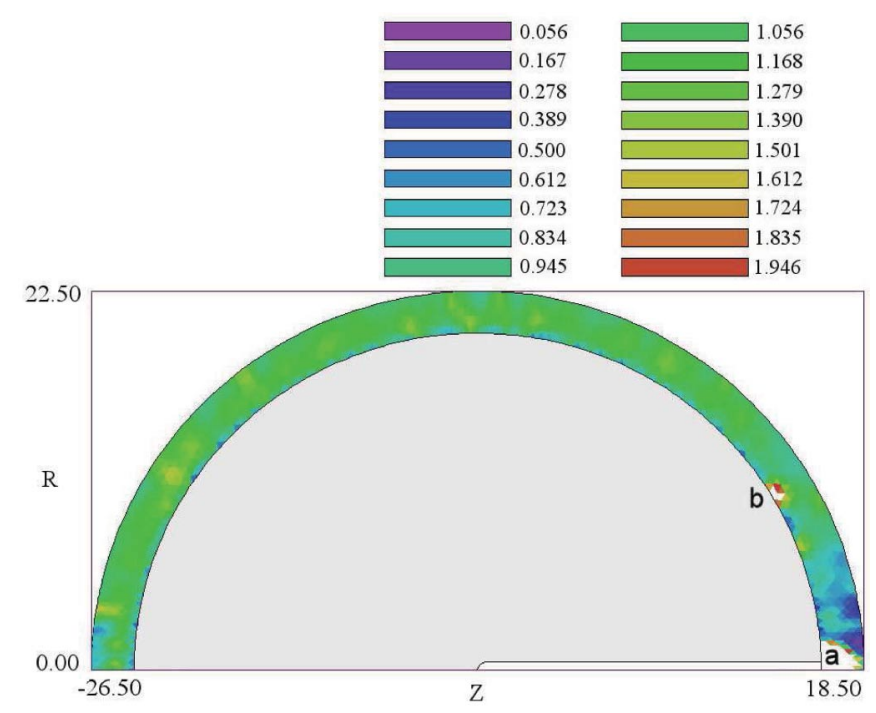

FIG. 11. (Color) Smoothed photon dose rate $(\mathrm{Gy} / \mathrm{s})$ in the test volume, CRAD diode benchmark simulation. Multiply values by $10^{9}$. with long drift orbits. The hot spot marked $b$ was caused by an escaping positron. Eliminating these areas, we found that the volume-weighted average dose rate in the shell was $d_{a v}=1.174 \times 10^{9} \mathrm{~Gy} / \mathrm{s}$. Taking the average shell radius as $r_{a v}=21.25 \mathrm{~mm}$, the projected dose at $1 \mathrm{~m}$ for a pulse length $\Delta t$ is given by

$$
D=d_{a v} r_{a v}^{2} \Delta t
$$

For $\Delta t=30 \mathrm{~ns}$, Eq. (3) predicts a dose of $D=$ $0.0159 \mathrm{~Gy}$ or $1.59 \mathrm{rad}$ at $1.0 \mathrm{~m}$. This dose is sufficient for many pulse radiography applications.

\section{ACKNOWLEDGMENTS}

The authors are grateful to Timothy Houck and Fletcher Goldin for useful discussions regarding the CRAD experiments. This work was performed under the auspices of the U.S. Department of Energy by University of California, Lawrence Livermore National Laboratory under Contract No. W-7405-Eng-48.

[1] Proceedings of the 1984 Flash Radiography Symposium, edited by E. Webster and A. Kennedy (American Society for Nondestructive Testing, Columbus, OH, 1985).

[2] F. Jamet and G. Thomer, Flash Radiography (Elsevier Scientific Publishing Co., Amsterdam, 1976).

[3] C. Ekdahl, IEEE Trans. Plasma Sci. 33, 892 (2005).

[4] L. Multhauf, N. Back, L. Simmons, and J. Zentler, Proc. SPIE Int. Soc. Opt. Eng. 4948, 622 (2003).

[5] D. J. P. R. Menge, J. Maenchen, D. Rovang, B. Oliver, D. Rose, and D. Welch, Rev. Sci. Instrum. 74, 3628 (2003).

[6] P. R. Menge, D. L. Johnson, J.E. Maenchen, C. Olson, D. C. Rovang, D. Droemer, E. Hunt, B. V. Oliver, D. V. Rose, and D. R. Welch, Sandia National Laboratories Report No. SAND2002-0092, 2002 (unpublished).

[7] D. Weidenheimer, in Proceedings of the 13th IEEE International. Pulsed Power Conference, edited by R. Reinovsky and M. Newton (IEEE, Piscataway, New Jersey, 2001), Vol. 1, p. 591.

[8] W. B. Herrmannsfeldt, Stanford Linear Accelerator Center, Report No. SLAC-331, 1988 (unpublished).

[9] A. Paul, Lawrence Berkeley Laboratory Report No. LBL13241, 1982 (unpublished).

[10] D. Vogel, Lawrence Berkeley Laboratory Report No. LBL-18871, 1985 (unpublished).

[11] J. Boers, Sandia National Laboratories Report No. SAND 79-1027, 1980 (unpublished).

[12] R. True, IEEE Trans. Nucl. Sci. 32, 2611 (1985).

[13] R. True, in Computational Accelerator Physics, edited by R. Ryne (American Institute of Physics, New York, 1994), p. 493.

[14] S. Humphries, in Computational Accelerator Physics, edited by R. Ryne (American Institute of Physics, New York, 1994), p. 597.

[15] S. Humphries, in Beams 94: Proceedings of the 10th Conference on High-Power Particle Beams, edited by W. 
Rix and R. White (National Technical Information Service Report No. NTIS PB95-144317, 1994).

[16] S. B. Swanekamp, R. J. Commisso, G. Cooperstien, P. F. Ottinger, and J.W. Schumer, Phys. Plasmas 7, 5214 (2000).

[17] R. Commisso, G. Cooperstein, D. Hinshelwood, D. Mosher, P. Ottinger, S. Stephanakis, S. Swanekamp, B. Weber, and F. Young, IEEE Trans. Plasma Sci. 30, 338 (2002).

[18] S. Humphries, Charged Particle Beams (Wiley Interscience, New York, 1990).

[19] S. Humphries, Laser Part. Beams 18, 601 (2000).

[20] S. Humphries, J. Comput. Phys. 125, 448 (1996).

[21] T. Houck (private communication).

[22] J. Creedon, Physics International Company Report No. PIIR-17-72A, 1972 (unpublished).
[23] J. Smith et al., in Proceedings of the 14th International Conference on High Power Particle Beams, edited by T. A. Melhorn and M.A. Sweeney (American Institute of Physics, Melville, NY, 2002), Vol. 1.

[24] F. Salvat, J. Fernandez-Varea, and J. Sempau, in Proceedings of the Workshop/Training Course, OECD/ NEA, 2003 (unpublished).

[25] I. Kawrakow and D. Rogers, NRCC Report No. PIRS-701, 2001 (unpublished).

[26] J. Halbleib, Sandia National Laboratories Report No. SAND91-1634, 1992 (unpublished).

[27] A. S. Group, Report No. CERN, W5013, 1994 (unpublished).

[28] X-5 Monte Carlo Team, Los Alamos National Laboratory Report No. LA-UR-03-1987, 2004 (unpublished). 